\title{
Diagnosis of congenital/perinatal infections by neonatologists: a national survey
}

\author{
Jung S. Hwang ${ }^{1} \cdot$ Scott Friedlander ${ }^{2} \cdot$ Virender K. Rehan ${ }^{1,2} \cdot$ Kenneth M. Zangwill ${ }^{1,2}$
}

Received: 18 December 2018 / Revised: 20 February 2019 / Accepted: 5 March 2019 / Published online: 26 March 2019

(c) Springer Nature America, Inc. 2019

\begin{abstract}
Objective To describe the clinical approach used by neonatologists for diagnosis of congenital/perinatal infections (CPI); no such data currently exist.

Study design A national survey regarding the diagnosis of toxoplasma, syphilis, rubella, cytomegalovirus, and herpes simplex virus (HSV) infection in neonates.

Result We received $553(11 \%)$ responses. Central nervous system calcification or hydrocephalus was the commonest trigger to pursue a CPI diagnosis (98\%); maternal history was the least frequent $(67 \%)$. Four hundred twenty-two $(76 \%)$ used general screening such as "TORCH titer screen" (57\%) or total IgG or IgM (39\%). Further evaluation targeted known clinical sequelae; but cerebrospinal fluid testing was used in only $65 \%$ of those suspected of having HSV or syphilis. Fiftysix percent chose a treponemal instead of a non-treponemal test for syphilis. Multivariable analyses did not identify factors associated with the clinical diagnostic approach.

Conclusion We observed clinically important deviations from CPI diagnostic test recommendations in a national cohort of neonatologists.
\end{abstract}

\section{Introduction}

Congenital/perinatal infection (CPI) continues to result in significant clinical and economic consequences to individual patients and society [1-4]. These infections, sometimes referred to by the acronym "TORCH", classically include toxoplasmosis, syphilis, rubella, cytomegalovirus (CMV), and herpes simplex virus (HSV). Congenital rubella was declared eliminated from the US in 2004 [5] although rare importations may occur as many countries do not have routine rubella vaccinations [6]. Other congenitally transmitted viral infections may present similarly such as

Supplementary information The online version of this article (https:// doi.org/10.1038/s41372-019-0364-3) contains supplementary material, which is available to authorized users.

Kenneth M. Zangwill

kzangwill@labiomed.org

1 Department of Pediatrics, Harbor-UCLA Medical Center, Torrance, CA, USA

2 Los Angeles Biomedical Research Institute, Harbor-UCLA Medical Center, Torrance, CA, USA enterovirus, parvovirus, varicella virus, and Zika virus [7], among others. The potential long-term morbidity of CPI is infection-specific, but may include a broad range of morbidities such as blindness, hearing loss, developmental delay, and neurologic disability, including death [8-10].

Neonatologists frequently care for newborns with signs and symptoms consistent with CPI. As such, it is important that clinicians are aware of current recommendations regarding the diagnostic approach for each. Early postnatal diagnosis improves overall outcome by enabling timely initiation of specific therapy and other interventions to minimize the likelihood of acute and/or long-term damage. Despite the frequency with which evaluation for CPI is performed, there are no national data on actual clinical decision-making on this topic. We performed a national survey of neonatal practitioners to evaluate diagnostic practices utilized for neonates suspected of having CPI.

\section{Methods}

We developed an anonymous survey instrument to identify decision-making and practice behavior regarding the diagnosis of CPI among a diverse sample of neonatal 
Table 1 Recommended definitive diagnostic testing for congenital/perinatal infections in neonates in July/August 2017

\begin{tabular}{|c|c|c|}
\hline Congenital/perinatal infection & AAP Red Book [11] & $\mathrm{CDC}^{\mathrm{a}}$ \\
\hline Toxoplasma & $\begin{array}{l}\text { (a) IgG, IgM, and IgA antibody } \\
\text { (b) PCR of CSF, blood, or urine }\end{array}$ & No specific recommendation for neonates. \\
\hline Syphilis & $\begin{array}{l}\text { (a) Blood RPR or VDRL and CSF VDRL, if } \\
\text { clinically indicated. } \\
\text { "Some experts" recommend FTA-ABS of CSF } \\
\text { over CSF VDRL. }\end{array}$ & $\begin{array}{l}\text { Nontreponemal test (RPR/VDRL). Treponemal testing "not } \\
\text { recommended". }\end{array}$ \\
\hline Rubella & $\begin{array}{l}\text { (a) IgM antibody } \\
\text { (b) Throat or nasal swab culture/PCR }\end{array}$ & IgM antibody \\
\hline Cytomegalovirus & Tissue or blood culture or PCR & $\begin{array}{l}\text { PCR of urine, saliva (preferred), or blood. } \\
\text { "[CMV] cannot be diagnosed using tests that detect } \\
\text { antibodies". }\end{array}$ \\
\hline Herpes simplex virus & $\begin{array}{l}\text { (a) Culture or PCR of surface specimens, skin, } \\
\text { tissue, CSF, or blood. } \\
\text { (b) Direct fluorescent antibody tests "are available". } \\
\text { "Serologic testing is not useful in neonates". }\end{array}$ & No specific recommendation for neonates. \\
\hline
\end{tabular}

practitioners in the US. The questions were separated into three general categories: (1) clinical triggers that lead to initial consideration of CPI; (2) preferred pathogen-specific supportive diagnostic testing; and (3) test choices for definitive diagnostic confirmation of specific CPIs. These categories reflect an attempt to probe the real world clinical reality in which a set of clinical circumstances may trigger a potential CPI diagnosis (e.g., rash or a positive maternal rapid plasma reagin [RPR]), which may be followed by collection of CPI-specific data (e.g., long bone radiography for syphilitic osteitis) and thereafter definitive testing to identify the etiologic agent. We appreciate that these may overlap in time as well. We asked respondents to assume, for the purposes of this survey, that all available diagnostic options were available at their institution. We also collected demographic, and practice-related information as well as clinical experience with CPI from each respondent.

The survey included 18 questions and was distributed via SurveyMonkey (www.surveymonkey.com). The final survey incorporated changes following a pilot study of 11 neonatologists and fellows in neonatal-perinatal medicine at two separate institutions. Prior to the pilot, we also received feedback from the American Academy of Pediatrics (AAP) Section on Neonatal Perinatal Medicine (SONPM) without, however, its overt endorsement of this research activity. Respondent contact information was compiled from publicly available lists on the AAP and SONPM member roster and the general AAP website followed by elimination of duplicates.

The survey was performed in July and August 2017 and included a brief introduction explaining the purpose of the study in the first contact (see Appendix). Four weekly reminders were sent via SurveyMonkey to non-responders only. The survey was closed 8 weeks from the initial contact attempt. This study was deemed Exempt by the John F. Wolf Human Subjects Committee at Los Angeles Biomedical Research Institute at Harbor-UCLA Medical Center.

\section{Statistical analysis}

We performed descriptive analyses of physician data including years of practice, classification of their primary work unit, geographic location, and other variables. Further, physician responses pertaining to specific diagnostic testing for each infection type were dichotomized to represent either a "recommended" or an "incorrect" choice based on contemporaneous (July/August 2017) best practice standards as noted by the AAP Committee on Infectious Diseases (Red Book, 30th Edition, 2015) and/or the U.S. Centers for Disease Control and Prevention [11, 12]. For neonatal syphilis, CMV, and rubella, the recommendations were very similar between AAP and CDC. Responses consistent with either source were considered "recommended". For HSV and toxoplasma, CDC did not make an explicit recommendation for neonates (Table 1). We defined the recommended definitive diagnostic test(s) as follows: (1) HSV polymerase chain reaction (PCR) [blood, cerebrospinal fluid (CSF), or skin] or culture [blood, CSF, skin or nose, throat, eye, rectum], direct fluorescent antibody test; (2) CMV (PCR [blood, CSF, or urine] or culture [blood, CSF, urine, saliva]); (3) rubella (culture of throat, nasal swab or blood, IgM, or PCR [throat or nasal swab]); (4) toxoplasmosis [IgM or IgA, PCR blood or other tissue]; and syphilis (serum RPR or CSF Venereal Disease 
Research Laboratory [VDRL] or placental pathology). "Other, please specify" was an option for 10 of the 18 questions. For questions that inquired of the definitive CPIspecific diagnostic testing options, "Other" was chosen for $\leq 1 \%$ for each CPI; these were therefore reviewed and not included in the final analysis. We did not attempt to dichotomize the clinical triggers and supportive testing questions (as recommended or not) since such decisionmaking is not as clearly defined in guidelines as are available for definitive etiologic testing.

We completed multivariable logistic regression analyses to evaluate potential predictors of individual diagnostic choices for the definitive diagnosis of each CPI. Models were performed for each pathogen to examine clinical, physician, and practice characteristic including geographic region, years of practice, classification of primary practice unit, whether an infectious disease (ID) specialist was consulted, and the number of patients with each CPI seen by the physician. All analyses were completed with Stata 14.2 with statistical significance defined as $p<0.05$.

\section{Results}

We received 553/4843 (11\%) unique responses. Respondents were well-distributed by practice location in the US and years in practice and $58 \%$ usually or always consult an ID specialist around the diagnosis of CPI (Table 2). Several nonspecific clinical findings, when occurring in isolation, were queried to identify those that serve as clinical triggers leading to further testing for CPI. All were known features of various CPIs; the most common triggers were the presence of incidental central nervous system (CNS) calcifications or isolated hydrocephalus (98\%), microcephaly (91\%), or cataracts or other eye findings (91\%). Slightly less commonly chosen were hepato- or splenomegaly and/or jaundice (84\%) and neonatal skin lesions (78\%). The least frequently chosen options were the presence of intrauterine growth restriction $(69 \%)$ or an abnormal maternal prenatal laboratory test or the presence of a prenatal sexually transmitted disease (67\%).

After screening, if the clinician was "seriously considering" a CPI, 420/552 (76\%) reported use of a general approach that was not pathogen-specific (Fig. 1). The most commonly chosen tests were cranial imaging (61\%) and a "TORCH titer screen" (57\%). Total IgG or IgM testing was chosen by $215 / 552$ (39\%) of neonatologists. Multivariable analyses did not identify any specific physician or practice characteristic that predicted either (1) specific clinical triggers that resulted in a decision to perform initial screening for CPI or (2) choice of a particular non-pathogen-specific screening test.

Testing to support the presumptive diagnosis of specific CPIs varied by pathogen (Table 3 ). Choices were generally
Table 2 Demographic and practice characteristics of respondents

\begin{tabular}{|c|c|}
\hline Variable & Number $(\%)^{\mathrm{a}}$ \\
\hline \multicolumn{2}{|l|}{ Region } \\
\hline Northeast & $156(28.4)$ \\
\hline Midwest & $127(23.0)$ \\
\hline South & $132(24.0)$ \\
\hline West & $111(20.2)$ \\
\hline Other & $24(4.4)$ \\
\hline \multicolumn{2}{|c|}{ Do you consult an infectious disease specialist? } \\
\hline Always & $105(19.1)$ \\
\hline Usually & $212(38.6)$ \\
\hline Sometimes & $184(33.5)$ \\
\hline Rarely/Never & $38(6.9)$ \\
\hline N/A & $11(2.0)$ \\
\hline \multicolumn{2}{|l|}{ Years in Practice } \\
\hline$<5$ & $123(22.3)$ \\
\hline $5-9$ & $95(17.2)$ \\
\hline $10-14$ & $74(13.4)$ \\
\hline $15-19$ & $46(8.4)$ \\
\hline $20-29$ & $125(22.7)$ \\
\hline $30+$ & $88(16.0)$ \\
\hline \multicolumn{2}{|l|}{ Practice Type } \\
\hline Academic Center & $230(41.7)$ \\
\hline Academic Private Mix & $138(25.1)$ \\
\hline Private Practice & $147(26.7)$ \\
\hline Other & $36(6.5)$ \\
\hline \multicolumn{2}{|c|}{ Classification of Primary Care Unit } \\
\hline Level IV regional NICU & $206(37.4)$ \\
\hline Level III NICU & $295(53.5)$ \\
\hline Level I or II & $50(9.1)$ \\
\hline
\end{tabular}

${ }^{\mathrm{a}}$ Counts do not total 553 for all variables due to missing data

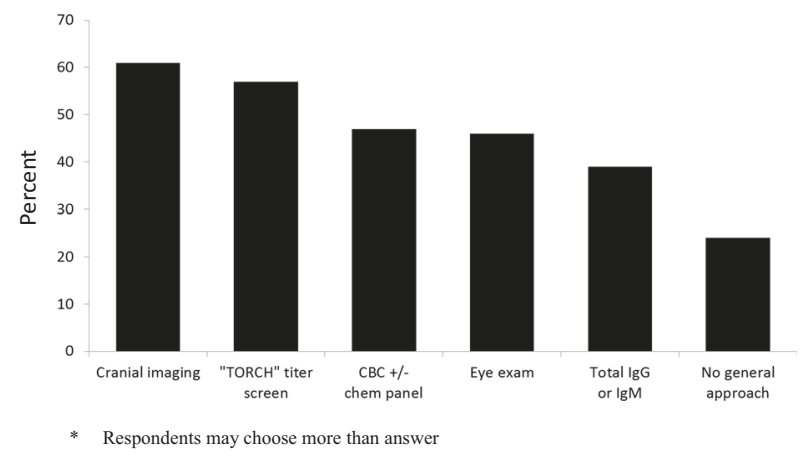

Fig. 1 General approach to diagnose congenital/perinatal infection if "seriously considering" diagnosis

targeted to the most common and expected sequelae for each CPI. Some recommended tests, however, were not performed for organ systems commonly affected. For example, CSF testing was performed in only $65 \%$ of infants suspected of having HSV or syphilis. Clinicians chose to 
Table 3 Supportive testing for congenital/perinatal infection by respondents

\begin{tabular}{llllll}
\hline Test $^{\mathrm{a}}$ & $\begin{array}{l}\text { HSV } \\
(n=552)\end{array}$ & $\begin{array}{l}\text { CMV } \\
(n=550)\end{array}$ & $\begin{array}{l}\text { Toxoplasma } \\
(n=548)\end{array}$ & $\begin{array}{l}\text { Rubella } \\
(n=542)\end{array}$ & $\begin{array}{l}\text { Syphilis } \\
(n=545)\end{array}$ \\
\hline Ophthalmologic exam & 16 & 56 & 62 & 60 & 24 \\
AST/ALT/bilirubin & 65 & 49 & 42 & 35 & 43 \\
Extremity radiograph & 2 & 2 & 2 & 9 & 55 \\
Cranial ultrasound & 11 & 65 & 69 & 27 & 12 \\
Skull radiograph & 2 & 4 & 4 & 2 & 7 \\
Head CT & 2 & 10 & 16 & 4 & 2 \\
CBC with differential & 44 & 51 & 41 & 41 & 48 \\
Hearing test & 7 & 54 & 20 & 30 & 11 \\
CSF testing & 65 & 15 & 23 & 9 & 65 \\
Placental pathology & 16 & 20 & 29 & 15 & 31 \\
None & 2 & 2 & 3 & 12 & 3 \\
\hline
\end{tabular}

$H S V$ herpes simplex virus, $C M V$ cytomegalovirus

aTotals do not equal $100 \%$ as choosing $>1$ test was permissible. All values in $\%$ perform an ophthalmologic examination in $56 \%$ and $62 \%$ of patients with suspected CMV or toxoplasmosis, respectively. Cranial imaging was not performed in $21 \%$ and $11 \%$ of those with suspected CMV and toxoplasmosis, respectively. When performed, the preferred method to evaluate for CNS calcifications was ultrasound. Conversely, clinicians chose certain tests that would not be expected to have a high yield in the acute phase for diagnosis in the newborn and/or are not generally recommended. For example, a hearing test was chosen for $7 \%, 11 \%$, and $20 \%$ for HSV, syphilis, and toxoplasmosis, respectively. Interestingly, no supportive tests were chosen by $12 \%$ of respondents in support of the diagnosis of rubella; for all of the other CPIs it was $\leq 3 \%$. In multivariable analyses, the choice of supportive testing did not vary substantially with practice location, years in practice, intensive care unit type, or frequency of consulting ID specialists.

Greater than $95 \%$ of respondents chose a recommended diagnostic test as their first choice for all CPIs except for syphilis for which $56 \%$ chose a fluorescent treponemal antibody test (FTA). Over $98 \%$ chose a recommended test for all diseases as the first or second choice. Approximately $90 \%$ clinicians had seen $<5$ patients with either rubella or toxoplasmosis in their career and 55\% had seen $<5$ patients with syphilis (Fig. 2). Multivariable analyses did not reveal consistent findings on clinician's likelihood of choosing the appropriate definitive diagnostic test (see Supplementary Table).

\section{Discussion}

Herein, we provide the first information on clinical decision-making choices made by US neonatologists as they evaluate neonates suspected of having CPI. We

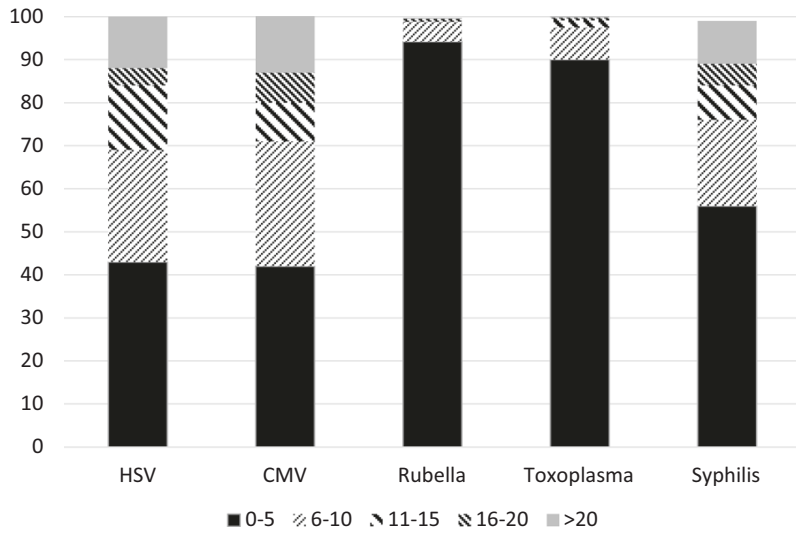

Fig. 2 Number of confirmed infections seen by respondents

evaluated clinical triggers for consideration of CPI and subsequent choices used to garner supportive evidence of disease and to definitively confirm infection. We found substantial variation among providers with regard to clinical triggers and supportive testing for these infections. Although the first choice for definitive testing was consistent with national recommendations (except for syphilis), there was also a common use of certain screening tests which are not generally recommended given their poor a priori test performance (i.e., total non-pathogen-specific antibody level). Our findings suggest potentially useful areas of opportunity for outreach among clinicians.

The recognition of CPI and the process for its identification can be challenging. Clinical presentation may include non-specific physical examination and/or laboratory abnormalities commonly encountered in uninfected neonates. Further, assessing likelihood for each condition may be problematic as the population-based incidence of most CPIs in the US is unclear. Specifically, the US incidence of toxoplasmosis, CMV, and HSV has been estimated from 
data extrapolated only from regional surveillance systems [13-15] and only congenital syphilis and rubella are nationally reportable. This, and further uncertainty as to disease incidence at the regional or local level, combined with the potential for serious morbidity associated with CPI generally [8-10], may contribute to a more "broad-brush" approach to its diagnosis.

After identification of a clinical trigger evoking concern for CPI, we found that $\sim 75 \%$ of neonatologists use a general screening test thereafter, as opposed to a pathogen-specific approach. Cranial imaging was the most commonly chosen screening test and ultrasound by far the preferred modality likely because of its ease of use combined with avoidance of exposure to ionizing radiation. A large variety of CNS changes are associated with CPIs and for detection of intracranial calcifications, only one study has directly compared modern ultrasound and computed tomography (CT) in neonates [16]. Among 33 Brazilian patients with congenital toxoplasmosis, there was $94 \%$ concordance between ultrasound and CT, although the median interval between tests was 15 days (IQR 3-36 days). Although more sensitive for structural abnormalities, magnetic resonance imaging may be insensitive to calcium and often logistically difficult to perform in neonatal patients. Cranial ultrasound is widely available and provides useful information; it is, however, non-specific and may be technically limited in its capacity to visualize the entire brain [17].

Performing a "TORCH titer screen" was chosen by over half of the respondents as the preferred screening test after a CPI is suspected. This practice has a long history [18], but is of little demonstrable value as a screening tool given its poor diagnostic return [19] and is generally not recommended [20]. It has been frequently noted that serologic testing for CPIs is most efficient and cost-effective when individual CPIs are targeted with due consideration of pretest probability based on maternal and neonatal findings [21]. Of note, $70 \%$ of respondents screen for CPI if isolated IUGR/SGA is present, a majority of whom use "TORCH" screening. Serologic testing for this indication in isolation, however, is of little to no utility [22-24] as $<10 \%$ of cases of IUGR are caused by CPI, the most common being CMV [25]. Such screening is similarly unrevealing for isolated CNS imaging findings such as ventriculomegaly, echogenic foci, and subependymal cysts [26].

Nearly $40 \%$ of neonatologists screened for CPI with total IgG/IgM testing. We believe this to be historically based on IgM data from the 1960s and early 1970s, a time during which this test was most studied for bacterial infection and congenital rubella infection. Those data generally showed this test to be not sensitive or specific [27-30]. In the modern era of rubella elimination in the US [5], this test has a high false positive rate and a positive predictive value for CPI of $<45 \%$ [31]. CPI-specific IgM in the neonate is a uniformly recommended diagnostic option for rubella and toxoplasma and remains under study for syphilis [11, 13, 32, 33]. Total IgM, however, should not be performed in this setting.

Per Table 3, once a particular CPI was identified as most likely, supportive testing for that infection was generally consistent with recommendations and/or expected complications. We also identified relatively frequent use of some tests not expected to be revealing and underutilization of more generally recommended tests. For example, we found that CSF studies were not pursued in $\sim 1 / 3$ of babies with possible syphilis or $\mathrm{HSV}$ disease nor ophthalmologic examination in $\sim 40 \%$ of those with possible CMV or toxoplasma. Other reports, primarily from single centers, have also shown low rates of CSF testing for infants suspected having HSV or syphilis [32, 34-37]. Although minor differences among recommending bodies must be acknowledged, such testing is generally recommended most importantly because extension to the CNS or eye structures may indicate dissemination which impacts management and outcome [38-40]. Other omitted testing that we identified, although very likely to indicate abnormalities, may not directly impact treatment and some may consider it unnecessary (e.g., liver function testing in CMV disease [50\% chose this option]).

We also identified some apparent overutilization of supportive testing. Twelve percent of respondents performed hearing tests for syphilis. It is a well-known cause of late-onset hearing loss [41], but a systematic review on pediatric sensorineural hearing loss (SNHL) in congenital syphilis reported no infants with this finding [42]. CDC recommends testing during the acute phase and the AAP does not, and this is no doubt confusing to clinicians. Similarly, the occurrence of early-onset SNHL (especially in the absence of meningitis) with HSV is unclear [43]; if it occurs it is very rare. We did not address the clinical practices for CPI follow-up testing beyond the neonatal period as such long-term medical care is typically performed by primary care pediatricians or specialists, not neonatologists.

We found that the great majority of clinicians chose the recommended definitive diagnostic test as their first choice for each CPI. The exception was syphilis for which a treponemal test was the first choice in $>50 \%$. The (non-treponemal) RPR, and its magnitude in comparison to maternal levels is the accepted metric to confirm a diagnosis and/or initiate a clinical evaluation for supportive findings [12]. Although the use of a treponemal test is recommended by AAP and CDC for non-neonatal disease confirmation, it is not so with the initial diagnosis of congenital disease. In the neonatal population, the $\mathrm{IgG}$ treponemal test is maternal in origin and no commercially available $\operatorname{IgM}$ test is approved by the U.S. Food and Drug Administration [12]. 
We acknowledge certain limitations to our study. Our conclusions reflect self-reported data which may or may not mirror actual medical practice. Despite a response rate of $11 \%$, our data included $>550$ respondents and represent the only and largest survey of neonatologists on this topic. As well, our sample is generally representative based on comparison to membership data provided by SONPM and other limited neonatal workforce information published by the AAP [44]; there are no recently published data on the demographics of practicing neonatologists in the US. Despite this, the statistical power for the multivariate analysis regarding the choice of the definitive diagnostic test for each CPI cannot exclude a type II error insofar as relatively few respondents chose an incorrect test. As with all survey studies, interpretation of each question may not have been uniform among study respondents and unintended systematic bias may be present [45]. Our data collection instrument was not externally validated beyond the pilot and SONPM feedback noted in Methods. Moreover, it is possible a small percentage of our respondents include caregivers that may also be involved in evaluating for newborn CPI such as hospitalists and some general pediatricians. We solicited answers among a range of pre-selected choices, but we also offered an option for alternative answers via text string. We also could not determine if choices reflect the providers' personal opinion or potentially that of infectious disease consultants who may have been involved; nor did we know if such consultants were available. On average, respondents took $<10 \mathrm{~min}$ to complete the survey (per SurveyMonkey analyses); therefore, we are confident that references sources were not regularly or consistently consulted during its completion.

Acknowledgements The authors thank the neonatologists for their participation in the survey.

Author contributions All authors contributed substantially to this work in its design, implementation, and/or analysis.

\section{Compliance with ethical standards}

Conflict of interest The authors declare that they have no conflict of interest.

Publisher's note: Springer Nature remains neutral with regard to jurisdictional claims in published maps and institutional affiliations.

\section{References}

1. Neu N, Duchon J, Zachariah P. TORCH infections. Clin Perinatol. 2015;42:77-103.

2. Owusu-Edusei K Jr, Introcaso CE, Chesson HW. Hospitalization cost of congenital syphilis diagnosis from insurance claims data in the United States. Sex Transm Dis. 2013;40:226-9.

3. Gantt S, Dionne F, Kozak FK, Goshen O, Goldfarb DM, Park AH. et al. Cost-effectiveness of universal and targeted newborn screening for congenital cytomegalovirus infection. JAMA Pediatr. 2016;170:1173-80.

4. Donoval BA, Passaro DJ, Klausne JD. The public health imperative for a neonatal herpes simplex virus infection surveillance system. Sex Transm Dis. 2006;33:170-4.

5. Centers for Disease Control and Prevention (CDC). Elimination of rubella and congenital rubella syndrome-United States, 19692004. MMWR Morb Mortal Wkly Rep. 2005;54:279-82.

6. Al Hammoud R, Murphy J, Pérez N. Imported congenital rubella syndrome, United States, 2017. Emerg Infect Dis. 2018;24:800.

7. Moore C, Staples J, Dobyns W, Pessoa A, Ventura C, Da Fonseca $\mathrm{E}$, et al. Characterizing the pattern of anomalies in congenital zika syndrome for pediatric clinicians. JAMA Pediatr. 2017;171: 288-95.

8. Cohen BE, Durstenfeld A, Roehm PC. Viral causes of hearing loss: a review for hearing health professionals. Trends Hear. 2014;18:1-17.

9. Mets MB, Chhabra MS. Eye manifestations of intrauterine infections and their impact on childhood blindness. Surv Ophthalmol. 2008;53:95-111.

10. Korndewal MJ, Oudesluys-Murphy AM, Kroes AC, Van der Sande MA, De Melker HE, Vossen AC. Long-term impairment attributable to congenital cytomegalovirus infection: a retrospective cohort study. Dev Med Child Neurol. 2017;59:1261-8.

11. American Academy of Pediatrics. In: Kimberlin DW, Brady MT, Jackson MA, Long SS, editors. Red Book: 2015 Report of the Committee on Infectious Diseases. 30th ed. Elk Grove Village, IL: American Academy of Pediatrics; 2015.

12. Available from Centers for Disease Control and Prevention [Internet]. http://www.cdc.gov. Accessed 19 Feb 2019.

13. Maldonado YA, Read JS, Committee on Infectious Diseases. Diagnosis, treatment, and prevention of congenital toxoplasmosis in the United States. Pediatrics. 2017;139:e20163860

14. Kenneson A, Cannon MJ. Review and meta-analysis of the epidemiology of congenital cytomegalovirus (CMV) infection. Rev Med Virol. 2007;17:253-76.

15. Handel S, Klingler EJ, Washburn K, Blank S, Schillinger JA. Population-based surveillance for neonatal herpes in New York City, April 2006-September 2010. Sex Transm Dis. 2011;38: 705-11.

16. Lago EG, Baldisserotto M, Filho JRH, Santiago D, Jungblut R. Agreement between ultrasonography and computed tomography in detecting intracranial calcifications in congenital toxoplasmosis. Clin Radiol. 2007;62:1004-11.

17. Lanari M, Capretti MG, Lazzarotto T. Neuroimaging examination of newborns in vertically acquired infections. J Matern Fetal Med. 2011;24:117-9.

18. Sever JL. Are routine prenatal TORCH titers necessary? Clin Microbiol Newsl. 1983;5:95-7.

19. Leland D, French ML, Kleiman MB, Schreiner RL. The use of TORCH titers. Pediatrics. 1983;72:41-3.

20. Del Pizzo J. Focus on diagnosis. Pediatr Rev. 2011;32:537-42.

21. de Jong EP, Vossen AC, Walther FJ, Lopriore E. How to use neonatal TORCH testing. Arch Dis Child Educ Prac. 2013;98: 93-8.

22. Van der Weiden $\mathrm{S}$, de Jong EP, Te Pas AB, Middeldorp JM, Vossen AC, Rijken M, et al. Is routine TORCH screening and urine CMV culture warranted in small for gestational age neonates? Early Hum Dev. 2011;87:103-7.

23. Primhak RA. Screening small for gestational age babies for congenital infection. Clin Pediatr. 1982;21:417-20.

24. Khan NA, Kazzi SNJ. Yield and costs of screening growthretarded infants for torch infection. Am J Perinatol. 2000;17: 131-6.

25. Hendrix N, Berghella V. Non-placental causes of intrauterine growth restriction. Semin Perinatol. 2008;32:161-5. 
26. Abdel-Fattah SA, Bhat A, Illanes S, Bartha JL, Carrington D. TORCH test for fetal medicine indications: only CMV is necessary in the United Kingdom. Prenat Diagn. 2005;5:1028-31.

27. Alford CA, Schaefer J, Blankenship WJ, Straumfjord JV, Cassady G. A correlative immunologic, microbiologic and clinical approach to the diagnosis of acute and chronic infections in newborn infants. N Engl J Med. 1967;227:437-49.

28. Finkel A, Dent PB, Emrich WH, Gent M, Rahim MA. Intrauterine infection and cord immunoglobulin M II. Clinical analysis of infants with elevated cord serum immunoglobulin M. Can Med Assoc J. 1974;110:38-42.

29. Miller MJ, Sunshine PJ, Remington JS. Quantitation of cord serum IgM and IgA as a screening procedure to detect congenital infection: results in 5006 infants. J Pediatr. 1969;75:1287-91.

30. Alford CA, Foft JW, Blankenship J, Cassady G, Benton JW. Subclinical central nervous system disease of neonates: a prospective study of infants born with increased levels of IgM. J Pediatr. 1969;75:1167-78.

31. Matthews TG, O'Herlihy C. Significance of raised immunoglobulin $\mathrm{M}$ levels in cord blood of small-for-gestational-age infants. Arch Dis Child. 1978;53:895-8.

32. Morshed MG, Singh AE. Recent trends in the serologic diagnosis of syphilis. Clin Vac Immunol. 2014;22:137-47.

33. American Academy of Pediatrics. Rubella. In: Kimberlin DW, Brady MT, Jackson MA, et al., editors. Red Book: 2018 Report of the Committee on Infectious Diseases. Elk Grove: American Academy of Pediatrics; 2018. p. 705-11.

34. Wolfert S, de Jong EP, Vossen A, Zwaveling J, Te Pas A, Walther $F$, et al. Diagnostic and therapeutic management for suspected neonatal herpes simplex virus infection. J Clin Virol. 2011;51:8-11.

35. Wilson P, Sutton AG, Nassef C, Falato C, Jhaveri R. Persistent gaps in appropriate use of empiric acyclovir in neonates. Clin Pediatr. 2017;56:472-9.
36. dos Santos RR, Niquini RP, Bastos FI, Domingues RMSM. Diagnostic and therapeutic knowledge and practices in the Management of congenital syphilis by pediatricians in public maternity hospitals in Brazil. Int J Health Serv. 2017;33:e238-50.

37. Le Chevalier PM, Alessandri JL, Traversier M, Cuillier F, Robin S, Ramful D. Evaluation of the management of pregnancies and infants at risk for congenital syphilis: La Réunion, 2008 to 2014. J Perinatol. 2017;37:116-21.

38. Villena I, Ancelle T, Delmas C, Garcia P, Brezin A, Thulliez P, et al. Congenital toxoplasmosis in France in 2007: first results from a national surveillance system. Eurosurveillance. 2010;15:19600.

39. Coats DK, Demmler GJ, Paysse EA, Du LT, Libby C, Congenital CMV, Longitudinal Study Group. Ophthalmologic findings in children with congenital cytomegalovirus infection. J Am Assoc Pediatr Ophthalmol Strab. 2000;4:110-6.

40. Kimberlin DW. Herpes simplex virus infections of the central nervous system. Sem Pediatr Infect Dis. 2003;14:83-9.

41. Fiumara NJ, Lessell S. Manifestations of late congenital syphilis. An analysis of 271 patients. Arch Dermatol. 1970;102:78-83.

42. Chau J, Atashband S, Chang E, Westerberg BD, Kozak FK. A systematic review of pediatric sensorineural hearing loss in congenital syphilis. Int J Pediatr Otorhinolaryngol. 2009;73:787-92.

43. Westerberg BD, Atashband S, Kozak FK. A systematic review of the incidence of sensorineural hearing loss in neonates exposed to herpes simplex virus (HSV). Int $\mathrm{J}$ Pediatr Otorhinolaryngol. 2008;72:931-7.

44. Althouse LA, Stockman JA. Pediatric workforce: a look at neonatal-perinatal medicine data from the American Board of Pediatrics. J Pediatr. 2006;148:727-9.

45. Kelley K, Clark B, Brown V, Sitzia J. Good practice in the conduct and reporting of survey research. Int J Qual Health Care. 2003;15:261-6. 